\title{
The appearance of two lock-in states in the vortex flow around an in-line forced oscillating circular cylinder
}

\author{
Y. Yokoi ${ }^{1, \mathrm{a}}$ and K. Hirao ${ }^{2}$ \\ ${ }^{1}$ National Defense Academy, Department of Mechanical Engineering, 1-10-20 Hashirimizu, Yokosuka 239-8686, Japan \\ ${ }^{2}$ Japan Air Self-Defense Force, Iruma Base, 2-3 Inariyama, Sayama, Saitama 350-1324, Japan
}

\begin{abstract}
In this study, the flow features of vortex shedding from a circular cylinder forced-oscillating in the in-line direction were investigated by use of flow visualization experiment and numerical simulation at the Reynolds number $R e=620$, with varied amplitude ratio and varied frequency ratio. As a result of the experiments, it was found that although the flow structure around the circular cylinder is two-dimensional in the lock-in state of simultaneous vortex shedding, the large scale three-dimensional instability is observed in the lock-in state of alternate vortex shedding through a time lag in the boundary layer separation along the cylinder span. As a result of calculations, two typical flow patterns of the lock-in were shown, and it was confirmed that the calculated flow patterns were in reasonable agreement with previous experimental results. The fluid force act on the oscillating cylinder was investigated. It was clarified that the amplitude of the lift coefficient was larger than the amplitude of the drag coefficient in the lock-in of alternate vortex shedding, and the amplitude of the drag coefficient was larger than the amplitude of the lift coefficient in the lock-in of simultaneous vortex shedding. When the amplitude ratio $2 a / d$ grows, this tendency becomes remarkable.
\end{abstract}

\section{Introduction}

The flow induced vibration problem is very important problem for engineering field so that there are many reports and useful knowledge has been accumulated by many researchers [1-4]. The "lock-in" is the phenomenon which the vortex shedding frequency from a cylinder synchronizes with the frequency of cylinder vibration. And it is one of most interesting phenomenon in the field of flow induced vibration. Although there are many reports about the flow pattern and the vortex shedding characteristic at the time of a lock-in in the report about a lock-in phenomenon, there are few reports about the mechanism of lock-in generating. In the flow pattern at the time of a lock-in, it is known that those flow patterns will be divided roughly into two kinds, an alternation type vortex shedding and a simultaneous type vortex shedding. However, there is almost no report investigated about the aspect of the flow of a region which were inserted into the two flow pattern regions and the aspect of transition of the vortex shedding configuration. In order to investigate the mechanism of lock-in generating, it becomes important to investigate the unsteady fluid force which the bluff cylinder receives. However, in an experiment, since it is fairly difficult to measure the true unsteady fluid force of the object which is oscillating, the investigation using a numerical simulation is expected. In recent years, the calculation environment of numerical computation has accomplished remarkable development. The count ability of the present personal computer is equipped with the count ability which exceeds an old supercomputer, and much useful calculation software is marketed. Then, the unsteady fluid force committed to the bluff cylinder by using calculation software as analysis tools with a commercial personal computer was estimated, and investigation of the mechanism of lock-in generating was tried.

In this study, the flow visualization experiment and the numerical simulation were performed. In the flow visualization experiment, it was investigated about a vortex shedding pattern, and its intermittence nature and three dimension nature of a vortex shedding. In the numerical simulation, it was investigated about the fluid force of the circular cylinder in the lock-in state at the time of the circular cylinder oscillation.

\section{Experimental apparatus and method}

\subsection{Outline of experimental apparatus}

The experimental apparatus consists of a towing water tank, a cylinder oscillating equipment, visualization equipment, and recording equipment. The towing water tank consists of a main tank unit, a rail unit, a towing carriage and drive equipment. The length of the main

\footnotetext{
a yokoi@nda.ac.jp
} 
tank unit is $10 \mathrm{~m}$, the width is $0.8 \mathrm{~m}$, the depth is $0.7 \mathrm{~m}$ and the both side walls are made from glass. So as not for the vibration when towing a carriage to propagate to the main tank unit, the main tank unit and rail unit become separate. The cover shot is shown in figure 1. The towing carriage which was put on the rail unit is operated by the drive equipment using two timing belts. The oscillator which installed CCD (charge-coupled device) camera and test cylinder, the visualization apparatus are loaded into the towing carriage. The cylinder oscillator is using a Scotch-yoke mechanism and the oscillator consists of a small-sized variable AC motor with a controller, a turn disk and a connecting rod. The amplitude of oscillation is set by changing the rotation radius of connecting edge on the turn disk, while the frequency of oscillation is controlled by changing the revolution speed of the turn disk. Two sets of the halogen lights of $500 \mathrm{~W}$ were used as a visualization lighting installation. Two sets of halogen light are equipped on the carriage of towing water tank. The recording equipment consists of a small 3CCD color video camera for industry, and a set of video recording playback equipment. The equipment on the towing carriage is shown in figure 2 .

\subsection{Test cylinder}

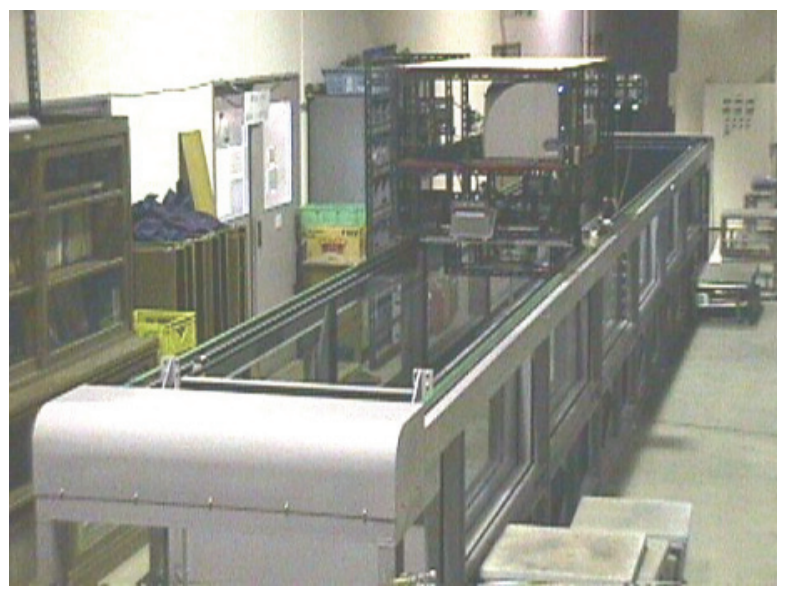

Fig. 1. Aspect of a towing water tank

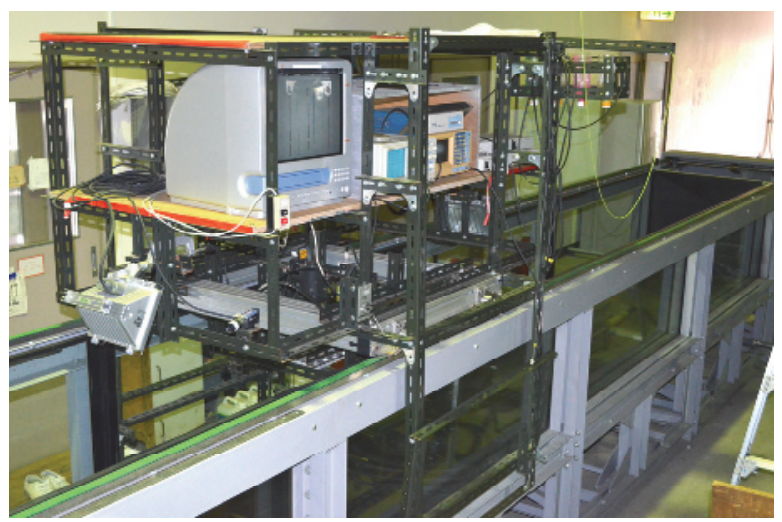

Fig. 2. Oscillating equipment and visualization observation equipment on a towing carriage
The test circular cylinder is made from acrylic resin with $20 \mathrm{~mm}$ of outside diameter, $16 \mathrm{~mm}$ caliber, $440 \mathrm{~mm}$ length. Dye ooze ports $(\varphi 1 \mathrm{~mm})$ for the flow visual observation are arranged from a front stagnation point to the position of \pm 60 degrees on the surface of the circular cylinder by two rows, and 50 and 51 ports are made respectively at intervals of $4 \mathrm{~mm}$. The row of the dye port becomes independent respectively, and can use the color of the tracer properly. An acrylic disk of $200 \mathrm{~mm}$ in the diameter with 30 degrees sharp edge is set up respectively at both ends of the circular cylinder span 400 $\mathrm{mm}$. The circular cylinder is installed in the oscillator by the horizontal posture through the holder arm to become $250 \mathrm{~mm}$ under the surface of the water when the water level in the test section of towing water tank is assumed to be $400 \mathrm{~mm}$, and oscillates periodically to the direction of the flow. The setting situation of the circular cylinder is shown in figure 3 .

\subsection{Flow visualization method}

The flow visualization was performed with the aid of dye injection method. The tracer inks of two colors that the specific gravity is adjusted ('Color-fan': turquoise and fluorescent pink) are oozed from the dye ports on the surface of cylinder. The arrangement of colors of the tracer inks is assumed that the surface of the water side is fluorescent pink, and the bottom of the water side is a turquoise (blue). Two halogen lights (white light $500 \mathrm{~W}$ ) were used for the lighting, and they were attached in the towing carriage front and rear. Two CCD video cameras were used to take a picture of the aspect of the flow. One was set up to look about the direction of the span, and other one was set up as seen axially. The visualized flow patterns were monitored by the CCD video cameras and recorded on video tapes.

\subsection{Experimental parameters}

The main experimental parameters were the oscillation frequency ratio $f / f_{K}$, the amplitude ratio $2 a / d$ and the mean flow velocity $U$. Here, the oscillation frequency ratio $f / f_{K}$ was defined by the ratio of circular cylinder

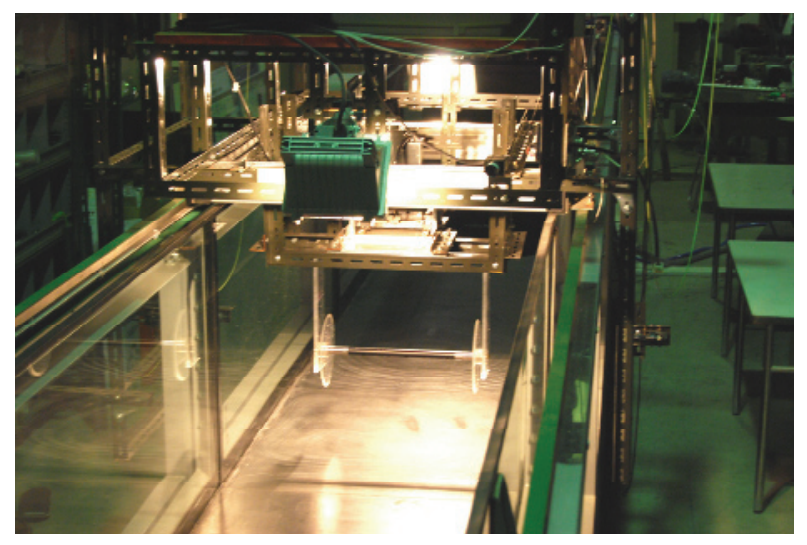

Fig. 3. The situation of the circular cylinder horizontally attached in the towing carriage through the holder arm 
oscillation frequency $f$ to natural Karman vortex's frequency $f_{K}$. The amplitude ratio $2 a / d$ is defined by the ratio of half amplitude of cylinder motion $a$ to the outside diameter of cylinder $d$. The mean flow velocity is the movement speed of the towing carriage of the towing water tank. The mean flow velocity was made into $0.04 \mathrm{~m} / \mathrm{s}$ so that it might be easy to perform visualization. The Reynolds number $R e$ based on the mean flow velocity $U$ and circular cylinder outer diameter $d$ was about $620(R e=U d / v$, where $v$ is the kinematic viscosity of water). In order to investigate replacement and intermittence nature of two kinds of vortex shedding patterns in the lock-in range, the amplitude ratio $2 a / d$ was set 0.2 , and the oscillation frequency ratio $f / f_{K}$ was varied every 0.1 steps from 1.6 to 2.6 .

\subsection{Outline of experimental procedure}

The experiment was performed by the following procedures. Water is filled so that the water level of water tank may be set to $400 \mathrm{~mm}$. The controller of towing drive is operated and the towing carriage velocity is set up. The test cylinder is installed on the towing carriage. The oscillation amplitude of the circular cylinder is set with the cylinder oscillator. And, circular cylinder oscillation frequency $f$ is gradually set based on the natural Karman vortex shedding frequency $f_{K}$ which is shed from stationary cylinder. Towing of carriage is performed after water fully suppressed. Two kinds of tracer ink for the flow visualization are oozed from the surface of the oscillating circular cylinder. The visualized flow features were monitored with two CCD video cameras, and recorded in video tapes. After towing, the carriage returns to the start position, changes a setup, and repeats operation. Here, in this experiment, the observation point of vortex shedding frequency was $3 d$ behind the cylinder. The vortex shedding frequency was obtained from the number and the measurement time of past vortex at the observation point. The cylinder oscillation frequency $f$ was calculated by measuring an oscillation cycle.

\section{Numerical calculations}

\subsection{Calculation apparatus and method}

The numerical experiment apparatus was consisted of simulation software and one notebook type computer (NEC; LaVie LL800/L) as calculation hardware which are on the market. The software which named 'UzuCrise 2D ver.1.1.3 rev.H (College Master Hands Inc., 2006)' was used. This software used the vortex method which is based on the Lagrangian analysis. Since the vortex method is the gridless method, it is suitable for the unsteady problem of such moving boundary.

The vortex method is a direct viscid-inviscid interaction scheme, and the emanation of velocity shear layers due to boundary layer separation is represented by introduction of discrete vortices with viscous core step by step of time. In the present study, the flow was assumed incompressible and two-dimensional flow field. The configuration of circular cylinder was represented 40 vortex panels using a boundary element method. The separating shear layers were represented the discreet vortices, which were introduced at the separation points. The mathematical base, the recent development of vortex method are explained in reference [5].

\subsection{Calculating condition and parameters}

The numerical experiment was performed at the twodimensional calculation for incompressible and viscous flow. The circular cylinder was divided into 40 panels which distributed the vorticity. Every calculation continued to more than non-dimensional time $T=200$. The main parameters of numerical experiment were the oscillation amplitude ratio $2 a / d$, the oscillation frequency ratio $f / f_{K}$ and the Reynolds number $R e$. The oscillation amplitude ratio $2 a / d$ is five kinds, and is $0.0,0.125,0.25$, $0.5,0.75$, and 1.0 , respectively. The oscillation frequency ratio $f / f_{K}$ is two kinds, and is the oscillation frequency ratios 1.6 and 3.0 in which an alternate vortex shedding lock-in and a simultaneous vortex shedding lock-in occur. The Reynolds number is 620 in one kind.

\section{Experimental results and discussion}

\subsection{Intermittence nature of two kinds vortex shedding}

The variations of mean vortex shedding frequency ratio $f_{V K} / f_{K}$ with oscillation ratio $f / f_{K}$ are shown in figure 4 . In the figure, the abscissa is the oscillation frequency ratio $f / f_{K}$ with the reduced velocity $\operatorname{Vr}\left(=U / f_{n} d\right.$, here $f_{n}$ is the natural frequency of the structure) and the ordinate is the vortex shedding frequency ratio $f_{V K} / f_{K}$. Two dotted lines in the figure have the ratio of the vortex shedding frequency in case of the cylinder oscillation $f_{V K}$ and the circular cylinder oscillating frequency $f$ and mean the

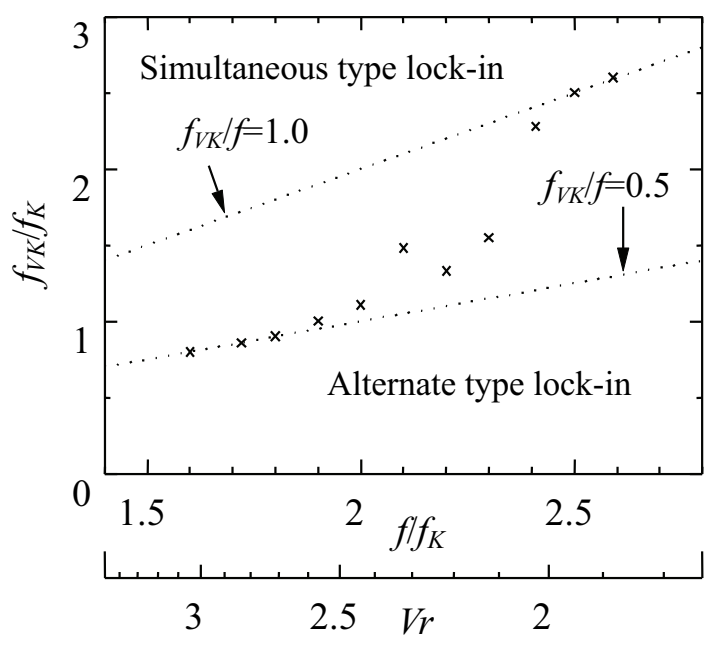

Fig. 4. Variation of mean vortex shedding frequency ratio $f_{V K} / f_{K}$ with oscillation frequency ratio $f / f_{K}$ 


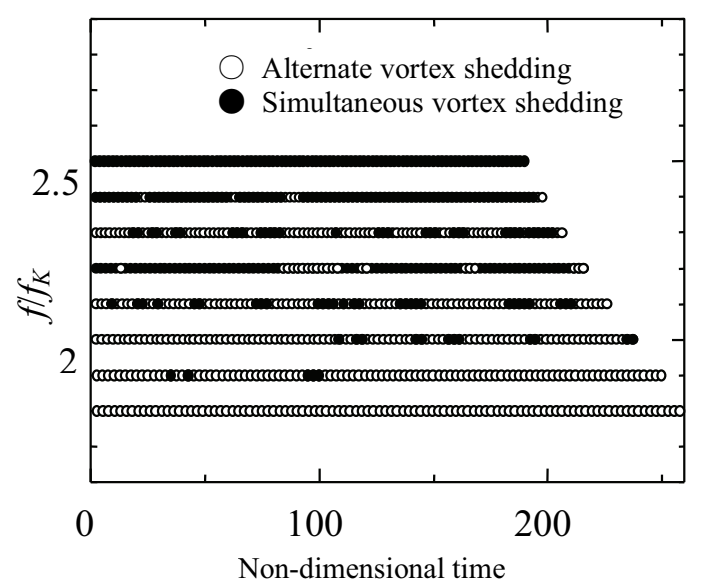

Fig. 5. Time histories of vortex shedding

"lock-in" status. Therefore, the occurrence of lock-in can be assumed when experiment data are shown on the line. When an oscillation frequency ratio $f / f_{K}$ is 1.8 or less, carrying out the lock-in of the vortex shedding frequency $f_{V K}$ to the frequency of the half of circular cylinder oscillation frequency $f$ is shown. It is imagined that the flow pattern at this time is an alternate vortex shedding type. On the other hand, when an oscillation frequency ratio $f / f_{K}$ is 2.5 or more, carrying out the lock-in of the vortex shedding frequency $f_{V K}$ to circular cylinder oscillation frequency $f$ is shown. It is imagined that the flow pattern at this time is a simultaneous vortex shedding type. Moreover, in the region (oscillation frequency ratio $f / f_{K}$ is from 1.9 to 2.4 ) from which it strayed off from the lock-in, it is expected that these two vortex shedding configurations appear intermittently.

An example of the time history of the vortex shedding is shown in figure 5. The observation position of a vortex shedding was the span central part of the oscillating circular cylinder, and observation followed 100 vortices, after running $2 \mathrm{~m}$ after towing. The white circle symbol and black painting circle symbol in the figure show the alternate vortex shedding and the simultaneous vortex shedding, respectively. Replacement of two kinds of vortex shedding configurations can be seen. The replacement is intermittent and it is found that there is no periodicity.

\subsection{Three-dimensional nature at the time of a vortex shedding}

The aspect of the boundary layer separation on a stationary circular cylinder is a three dimensional, and it is reported by the previous report [6] that the separation itself is unstable. In the case of the oscillating circular cylinder, the instability which changes with states of a lock-in was observed. The aspect of the vortex shedding from the circular cylinder in an alternate vortex shedding type lock-in state is shown in figure 6. Figure 6 (a) and (b) are the viewpoint of a bird's-eye view, and a viewpoint from the cylinder axis, respectively. Here, the tracer oozing interval from the cylinder surface is $2.25 d$, and was performed from five places. In figure 6 (a), the

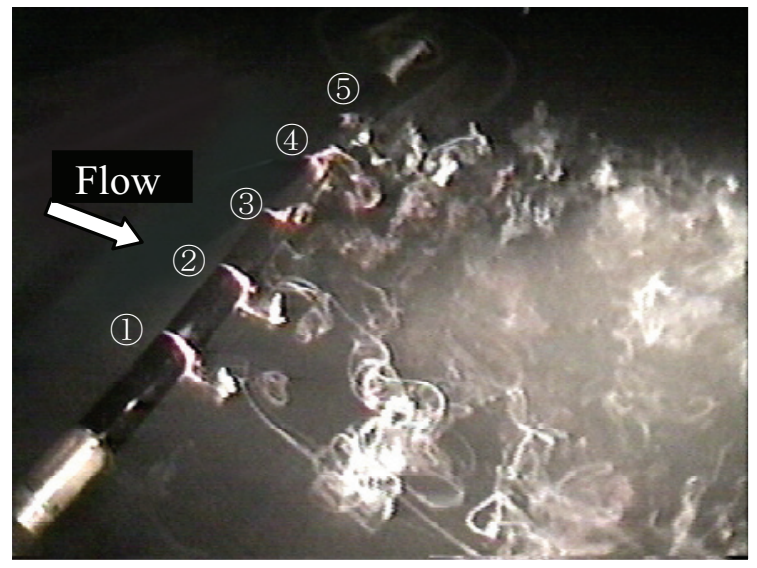

(a)

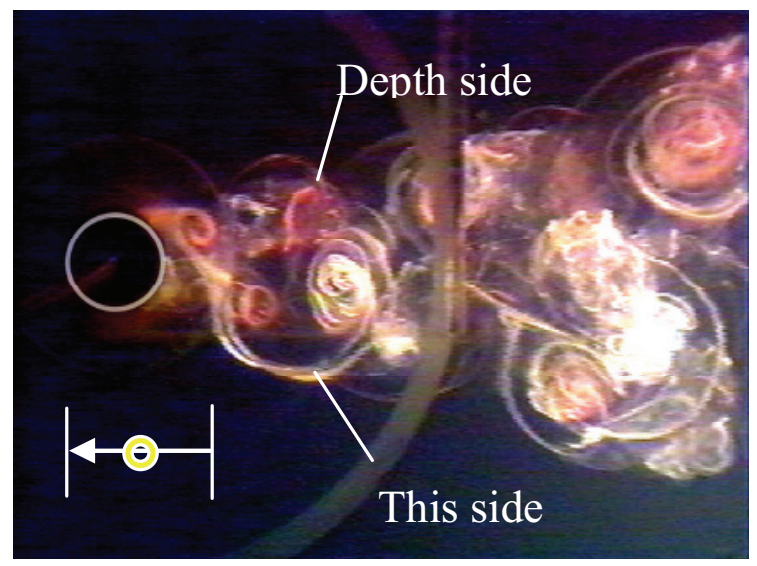

(b)

Fig. 6. Instantaneous flow pattern of lock-in state of $f_{V K} / f=0.5, R e=620, f \mid f_{K}=1.8$; (a) bird eye view, (b) side view

tracer oozing position was named 1, 2, 3, 4, and 5 from this side on account of explanation. The vortical roll rise has arisen from the cylinder bottom side in the tracer oozing positions 1 and 2. Contrary to it, the vortical roll rise has arisen from the cylinder top side in the tracer oozing positions 4 and 5. Those things show that there is a time lag in the direction of the cylinder span in the roll rise of the vortex at the time of vortex shedding. When the aspect of the flow is observed from the cylinder axis, existence of such a time lag is known clearly. The aspect of the flow observed from the circular cylinder axis is shown in figure 6 (b). The tracer streak lines of this side and the depth side are not in agreement, and it can be found that a time lag is in roll up of the vortex of an alternation type vortex shedding lock-in. Since strong two dimension nature ruled over by oscillation, it was observed that the boundary layer separation flow in the simultaneous vortex shedding type lock-in state does not have most fluctuation by the direction of cylinder span.

Figure 7 shows the aspect of the vortex shedding from the circular cylinder in the state where it strayed off from the lock-in. The tracer oozing position was named 1 , $2,3,4$, and 5 from this side on account of explanation here. Although it is the alternate vortex shedding in the position of 1 and 2, it is shown by the position of 3 that it is a simultaneous vortex shedding. It can understand that vortex shedding patterns differ for every part. This 


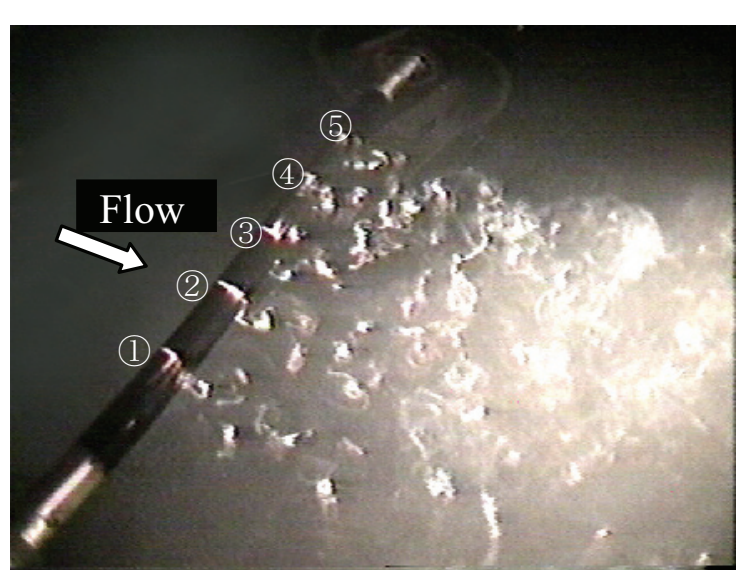

Fig. 7. Instantaneous flow pattern of non-lock-in state, $R e=620, f \mid f_{K}=2.3$

phenomenon is unsteady and the aspect of flow is changing with time progress. Therefore, when it observes about arbitrary circular cylinder sections, the phenomenon in which the vortex shedding of alternate vortex type or simultaneous vortex type puts in intermittently, and changes will be observed.

\section{Calculating results and discussion}

\subsection{In the case of stationary cylinder}

In order to verify the calculation software to be used, the experimental result and calculation result of a stationary circular cylinder were compared. The time history of the fluid force (a drag component and lift component) of acting on a stationary circular cylinder is shown in figure 8. The rapid increase in a drag by formation of an early twin vortex and generating of the lift by collapse of the symmetry by development of the twin vortex are shown. Moreover, oscillation of the drag lurking in periodic oscillation and lift oscillation of the lift produced by formation of a Karman vortex street is expressed. The relationship whose oscillation frequency of the drag is twice the oscillation frequency of the lift is shown. The following results were obtained after non-dimension time $T=100$. Here, the diagram which defines the quantity about the fluid force is shown in figure 9. The average value of drag coefficient was $C_{D A V E}=1.04$ and the mean amplitude of drag coefficient was $\mathrm{A}_{C D}=0.53$. The average value of lift coefficient was $C_{L A V E}=0.00$ and the route mean square (r.m.s.) of lift coefficient was $\mathrm{A}_{C L}=0.72$. The average Strouhal number for which it determined from the lift oscillating period $T$ was $S t_{A V E}=0.259$. The Strouhal number is defined by $S t=f d / U=d / \Delta t U=1 / T$. When the Reynolds number is $R e=620$, it is known that the experimental value of Strouhal number $S t$ is about 0.2 . Although it seems that this calculation result is highly calculated as compared with an experimental result, it seems that this calculation has obtained the comparatively good calculation result since a two dimensional calculation result becomes higher than an experimental result about 30 to $40 \%$. When the Karman vortex shedding frequency $f_{K}$ was calculated from the

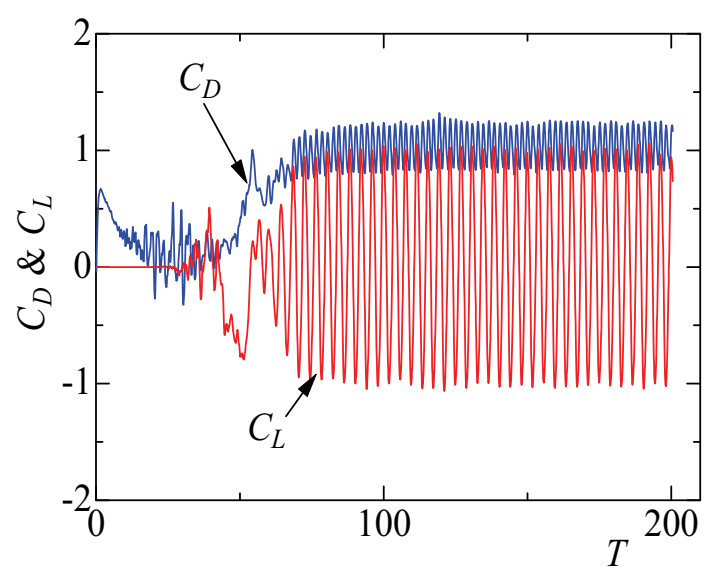

Fig. 8. Time histories of drag and lift coefficients

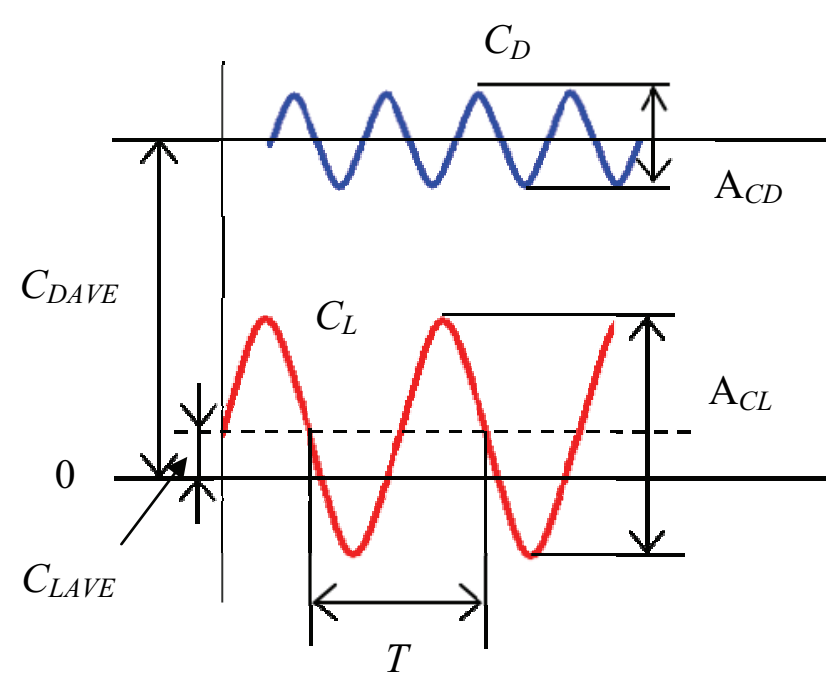

Fig. 9. The definition of the magnitude of the drag coefficient or the lift coefficient, and the definition of the oscillating period

average value of Strouhal number, the Karman vortex shedding frequency was $f_{K}=0.68 \mathrm{~Hz}$.

\subsection{In the case of oscillating cylinder}

When forced oscillation of the circular cylinder is carried out, it is one of the most interesting things of this study to investigate how fluid force changes. Although it is expected that the around flow of forced oscillation circular cylinder turns into a strong complicated flow of three dimensional nature, in the time of lock-in, it is described by the previous report [7] that two dimensional nature is maintained in the direction of cylinder span. So, it seems that it is applicable with the fluid analysis tools of the two dimension calculation technique. The numerical simulation was performed on the oscillating conditions which the alternate vortex shedding lock-in and the simultaneous vortex shedding lock-in generate. One example of the flow patterns obtained by calculation is shown in figure 10 . Here, the flow visualization 
photographs [8] in the similar experimental condition are shown for comparison. Figure 10 (a) is simulating the aspect of alternate vortex shedding lock-in. The vortex shedding direction changes and an aspect that the vortex shedding is performed alternately is shown. An aspect that the vortex discharged from the circular cylinder constitutes a vortex pair which differs in a rotatory direction, respectively, and the vortex street of mushroom shape like the section of a mushroom is formed is expressed well. Figure 10 (b) is simulating the aspect of a simultaneous vortex shedding lock-in. An aspect that the vortex of mushroom shape is simultaneously discharged from cylinder both sides, and the characteristic aspects of cylinder wake are shown. Since the characteristic flow pattern at the time of a lock-in is reproduced well, the credibility of the fluid force required in calculation can consider a high thing. Figure 11 shows the time history of a drag coefficient and a lift coefficient, and a circular cylinder position. The calculation varied amplitude ratio $2 a / d$ on the oscillating conditions used as an alternate vortex shedding lock-in and a simultaneous vortex shedding lock-in, and calculation was performed. Although based on oscillating conditions, it can be found that there is a section which drag amplitude increases and constitutes thrust force in instantaneously. Since the forced oscillation is carried out in the direction of a flow, the drag coefficient of an oscillating circular cylinder can be seen oscillating from the early stage synchronizing with cylinder oscillation. On the other hand, after carrying out time progress to some extent, oscillation of the lift coefficient of the oscillating circular cylinder can be seen start. The oscillating period of the lift coefficient at the time synchronizes the twice of the oscillating period of the drag coefficient. Moreover, in the alternate vortex shedding lock-in, the amplitude of the lift coefficient is larger than the amplitude of drag coefficient, and the amplitude of drag coefficient is larger than the amplitude of lift coefficient in the simultaneous vortex shedding lock-in. This phenomenon will become remarkable if oscillating amplitude ratio $2 a / d$ becomes large. It is guessed that the determination of the lock-in configuration is dependent on the magnitude of the amplitude of drag coefficient and its lift coefficient.

The mean amplitude of drag coefficient $A_{C D}$ and the mean amplitude of lift coefficient $\mathrm{A}_{C L}$ are shown in Table 1. In the oscillating state of alternate vortex shedding lock-in, the value of drag coefficient and the value of lift coefficient are larger than the value of stationary cylinder. And increase of amplitude ratio $2 a / d$ will tend to increase the value of drag coefficient, and the value of lift coefficient. On the other hand, in the oscillating state of simultaneous vortex shedding lock-in, the value of drag coefficient is large and the value of drag coefficient tends to increase with increase of amplitude ratio $2 a / d$. However, the value of lift coefficient will become smaller than the value of stationary cylinder case, and the force of oscillating to a flow and a perpendicular direction will be held down.

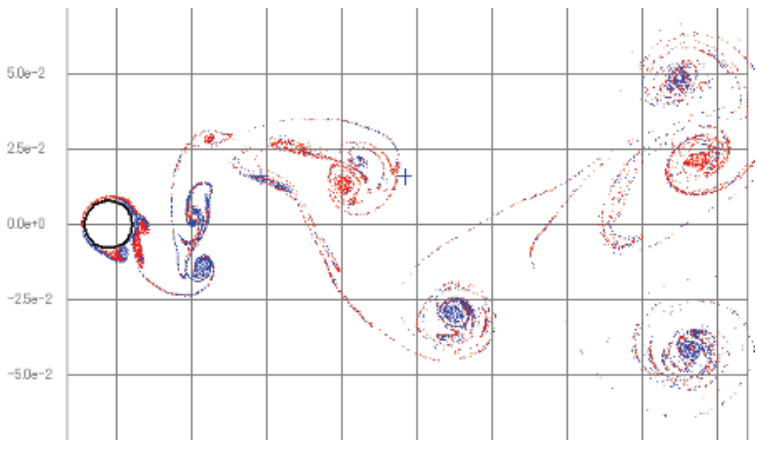

Alternate vortex shedding type lock-in

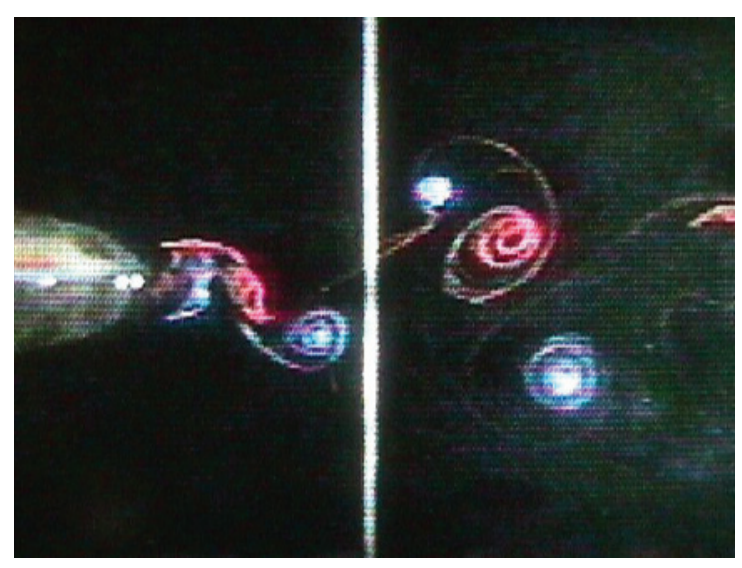

(a)

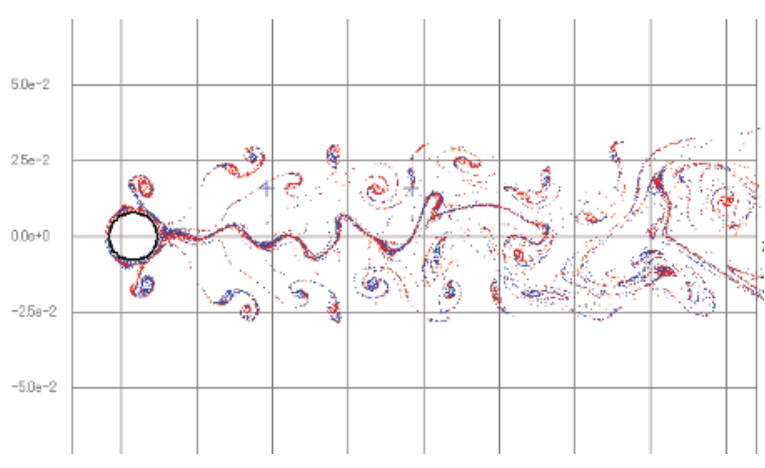

Simultaneous vortex shedding lock-in
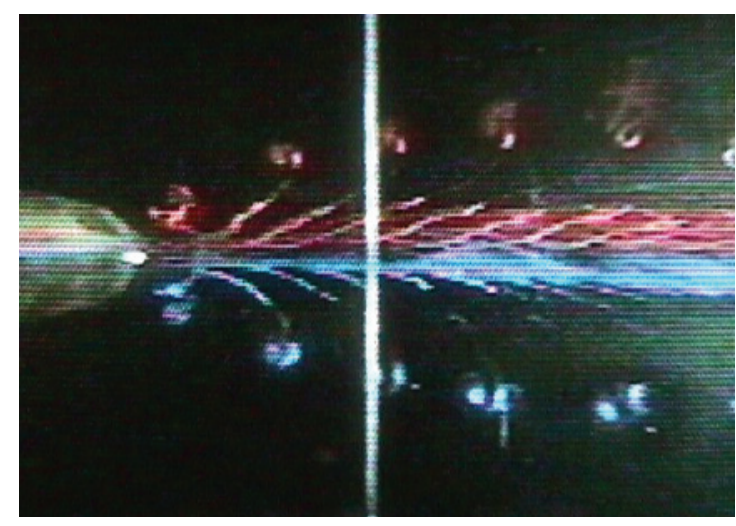

(b)

Fig. 10. Representative flow patterns in the cases of the calculation and the experiment [8]; (a) alternate vortex shedding lock-in case $\left(f_{V K} / f=1 / 2\right)$, (b) simultaneous vortex shedding lock-in case $\left(f_{V K} / f=1 / 1\right)$ 

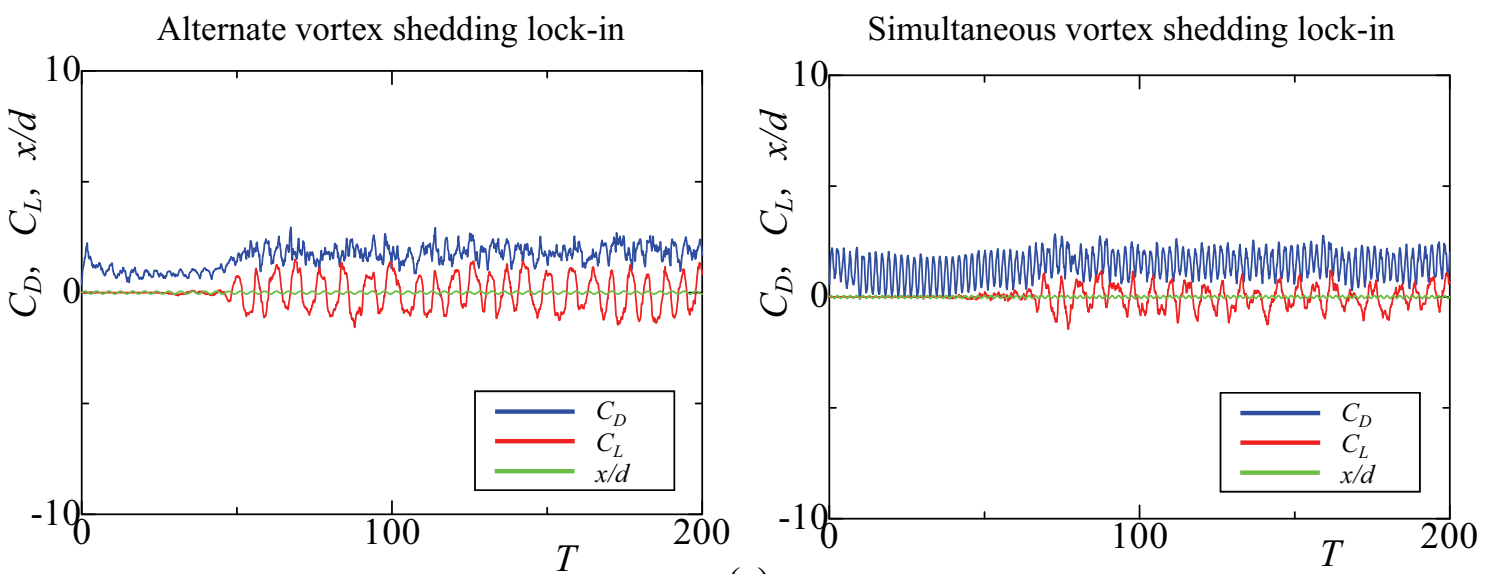

(a)
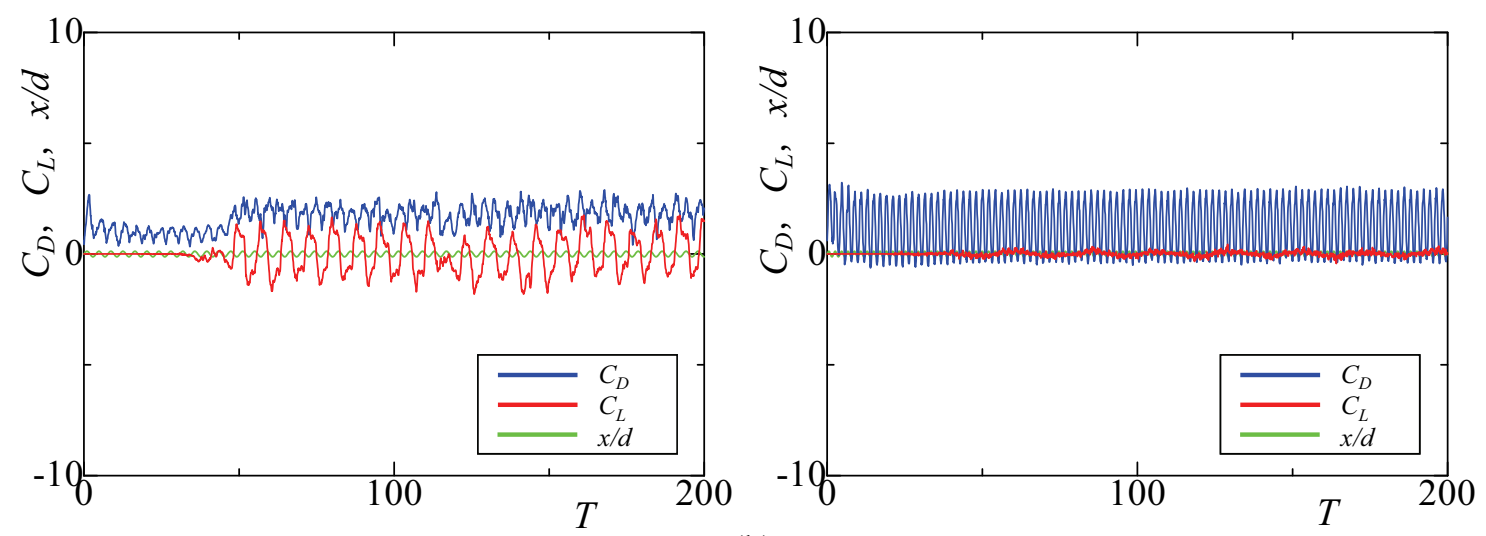

(b)
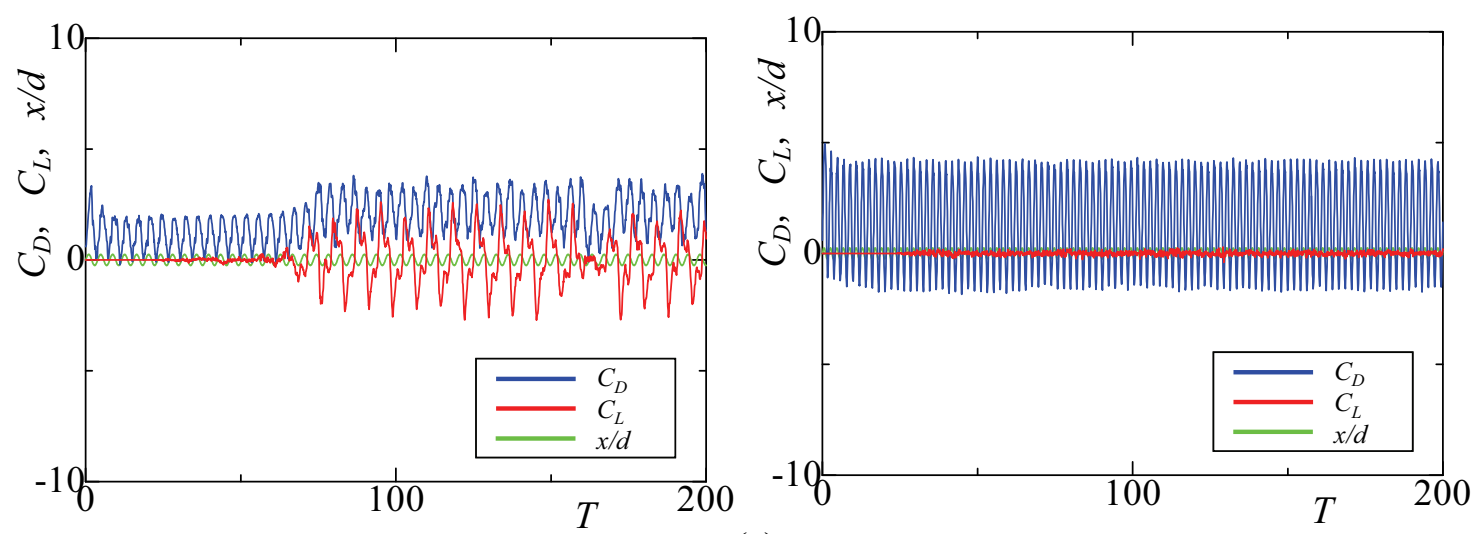

(c)
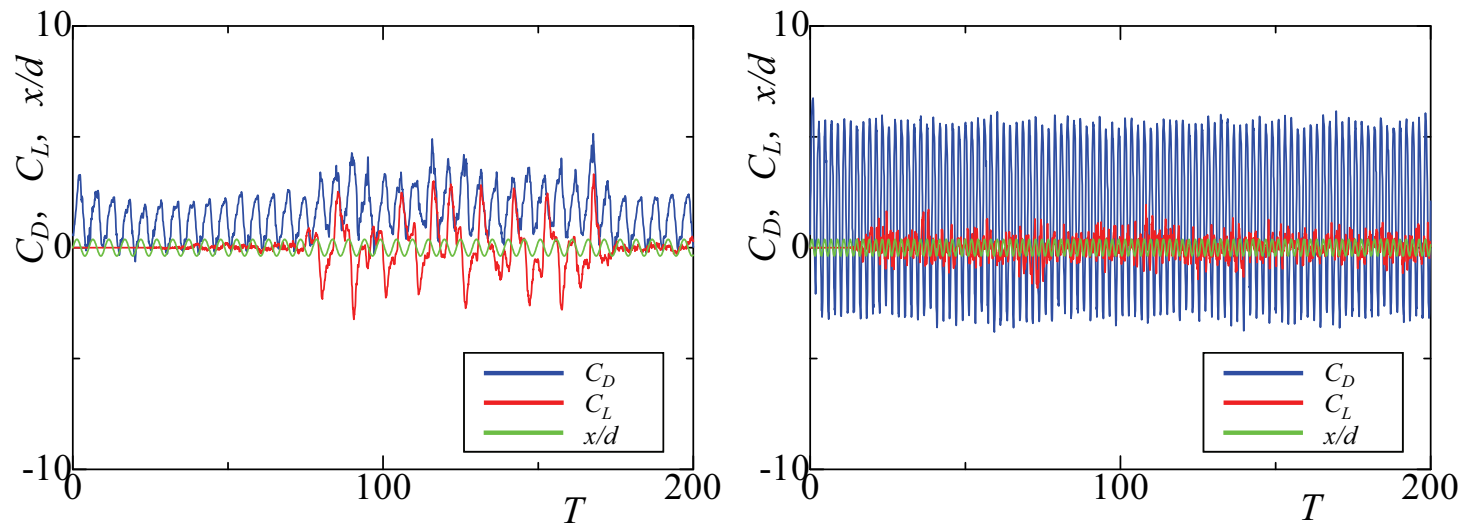

(d)

Fig. 11. Time histories of drag and lift coefficients and cylinder position; (a) $2 a / d=0.125$, (b) $2 a / d=0.25$, (c) $2 a / d=0.5$, (d) $2 a / d=0.75$ 
Table 1. Mean amplitude of drag and lift coefficients in the case of cylinder oscillation

\begin{tabular}{|c|c|c|c|c|c|c|c|c|c|c|}
\hline $2 a / d$ & \multicolumn{2}{|c|}{0.125} & \multicolumn{2}{|c|}{0.25} & \multicolumn{2}{|c|}{0.5} & \multicolumn{2}{|c|}{0.75} & \multicolumn{2}{|c|}{0.0} \\
\hline lock-in & $\mathrm{A}_{C D}$ & $\mathrm{~A}_{C L}$ & $\mathrm{~A}_{C D}$ & $\mathrm{~A}_{C L}$ & $\mathrm{~A}_{C D}$ & $\mathrm{~A}_{C L}$ & $\mathrm{~A}_{C D}$ & $\mathrm{~A}_{C L}$ & $\mathrm{~A}_{C D}$ & $\mathrm{~A}_{C L}$ \\
\hline $\begin{array}{l}\text { Alternate } \\
\left(f_{V K} / f=1 / 2\right)\end{array}$ & 1.4 & 2.16 & 1.56 & 2.28 & 2.51 & 4.08 & 2.67 & 3.83 & & \\
\hline $\begin{array}{l}\text { Simultaneous } \\
\left(f_{V K} / f=1 / 1\right)\end{array}$ & 1.75 & 1.61 & 3.39 & 0.36 & 5.82 & 0.36 & 9.00 & 1.14 & & \\
\hline
\end{tabular}

\section{Conclusions}

In this study, investigation about unsteady fluid force which acts on the aspect and circular cylinder of the vortex shedding from the circular cylinder which is carrying out the forced oscillation in the direction of the flow by the towing water tank experiment or the numerical simulation was performed. The following conclusions were obtained.

(1) The range of the oscillation frequency ratio from which it strays off from a complete lock-in state was 1.9 to 2.4. At this time, two kinds of vortex shedding configurations, an alternate vortex shedding and a simultaneous vortex shedding, should be intermingled, and they should interchange intermittently and appear.

(2) In the alternate vortex shedding lock-in state, a time lag should be in roll up of the vortex in the direction of cylinder span.

(3) In the simultaneous vortex shedding lock-in state, since strong two dimension nature rules over, there is no fluctuation of the direction of cylinder span. A twin vortex street is formed in the cylinder near wake, and it becomes an alternate vortex street in a far wake.

(4) The mechanism which carries out a lock-in was found by the numerical simulation.

(5) It was found that there are special sections which drag amplitude increases depending on oscillating conditions, and constitutes thrust in instantaneously.

(6) The result of numerical computation was good to the experimental value. Moreover, the flow pattern was also reproduced well. So, it is guessed that the calculated fluid force has high credibility. It was found that numerical computation is effective as a tool which estimates fluid force.

\section{References}

1. R.D. Blevins, flow-induced vibration (ROBERT E.KRIEGER PUBLISHING COMPANY), (1986)

2. O.M. Griffin, M.S. Hall, Trans. ASME J. Fluids Eng. 113, 526-537(1991)

3. A. Okajima, Trans. Jpn Soc. Mech. Eng. 65-635, 2190-2195(1999)

4. A. Okajima, Trans. Jpn Soc. Mech. Eng. 66-644, 948-953(2000)

5. M. Hafez, K. Oshima, Computational Fluid Dynamics Review 1995 (JOHN WIELY \& SONS), 334-353(1995)

6. Y. Yokoi, K. Kamemoto, JSME Int. J. Ser. II 35-2, 189-195(1992)

7. Y. Yokoi, K. Hirao, 7th World Conference on Experimental Heat Transfer, Fluid Mechanics and Thermodynamics 983-990(2009)

8. Y. Yokoi, T. Mashiba, J. Yamashita, Yamanashi District Conference Prepr. of Jpn. Soc. Mech. Eng. 71-72(2005) 